\title{
ESTUDIO DE LAS INNOVACIONES EMPRESARIALES NO SUSTENTADAS EN LA TECNOLOGÍA DE PUNTA ${ }^{1}$
}

\section{RESEARCH ON BUSINESS INNOVATIONS NOT BASED ON HIGH TECHNOLOGY ${ }^{1}$}

\section{Resumen}

El presente estudio fue realizado con el objetivo de estudiar y analizar si existen principios o factores guía, orientativos o aplicativos, que den lugar a las innovaciones que no están sustentadas en tecnología de punta. Luego de un proceso de recopilación de innovaciones no sustentadas en tecnología, se procedió a determinar cual es el factor no tecnológico de cada muestra; por último, se han agrupado las muestras en función a dichos factores o principios aglutinantes; relacionando cada conjunto agrupado con la literatura sobre el tema. Los resultados, habiendo analizado 342 unidades muestrales, comprenden la construcción de un número de cincuenta principios y siete grupos relacionados con las diversas corrientes teóricas del tópico.

Palabras clave: Innovación, tecnología, modelos de negocios, productos, principios heurísticos.

\begin{abstract}
Present work was developed with the aim of research and analize the existence of guidence, orientative or aplicative principles or factors, that lead to the development of innovations not based on high technology, After a not based technology innovations complitation process, it proceeded to determinate the non technological factor of each innovation sample. Finally, the samples were grouped in reference to the non technologycal factors or grouping principles; relating each grouping set to the topic literature. The results, having analized 342 samples, lead to the development of fifty principles and seven groups related to several theoretical currents.
\end{abstract}

Keywords: Innovation, technology, business models, products, heuristic principles.

CUNIS-MG. All rights reserved. 


\title{
1 INTRODUCCIÓN
}

\subsection{Problema de investigación}

En palabras de Benoit Godin:

\begin{abstract}
"...why did innovation come to be defined as technological innovation? Many people spontaneously understand innovation to be technological innovation. The literature itself takes this for granted. More often than not, studies on technological innovation simply use the term innovation, although they are really concerned with technological innovation. However, etymologically and historically, the concept of innovation is much broader." (Godin B, 2008).

“...¿¿por qué la innovación llegó a ser definida como innovación tecnológica? Muchas personas entienden espontáneamente a la innovación como innovación tecnológica. La literatura misma lo da por sentado. Muy a menudo, las investigaciones sobre innovación tecnológica utilizan solo el termino innovación, aunque se estén refiriendo a innovación tecnológica. Sin embargo, etimológica e históricamente, el concepto de innovación es mucho más amplio." (Godin B., 2008 - traducción del autor).
\end{abstract}

Aquí el autor se pregunta por qué la innovación llegó a ser definida como innovación tecnológica; para luego explicar y desarrollar en su trabajo de investigación, que el concepto de innovación es etomológica e históricamente, mucho más amplio que el de su relación con la tecnología.

Partiendo de las definiciones etimológicas, tanto en el diccionario de la lengua Inglesa CHAMBERS, como en en el diccionario de la Real Academia Española, se define innovar como el acto de introducir novedades que implican cambios "Mudar o alterar algo introduciendo novedades" (Diccionario Real Academia Española); "to make changes; to introduce new ideas, methods, etc" (Chambers Dictionary).

En relación al término tecnología, el diccionario de la Real Academia Española la define como el "Conjunto de teorías y de técnicas que permiten el aprovechamiento práctico del conocimiento científico" (RAE), y el diccionario Chambers como "the practical use of scientific knowledge in industry and everyday life" (Chambers Dictionary).

Ya desde su propia definción se advierte que la tecnología es susceptible de introducir cambios útiles (prácticos) y por ende provocar innovaciones. Por lo tanto, existe una entre la tecnología y la innovación. Sin embargo, cabe preguntarnos aquí si la tecnología es la única forma en que se alcanza una innovación; o lo que es lo mismo, responder a la pregunta: ¿Es la incorporación de tecnología, el único factor clave a efectos de innovar?

La OCDE (Organización de Cooperation para el Desarrollo Económico), desarrolla entre sus obras, dos manuales íntimamente relacionados con la medición de la ciencia, la tecnología y la innovación: el Manual de Frascati y el Manual de Oslo.

Mientras el Manual de Frascati expone las guía, orientaciones y consideraciones acerca de la medición de la Investigación y Desarrollo Experimental (I+D), medida que se ha utilizado para obtener los índices de intensidad tecnológica; el manual de Oslo es una guía para la recolección, los reportes y el uso de los datos sobre innovación.

Uno de los cambios más relevantes que se presentan en la tercera edición del manual de Oslo (versión del año 2005), es la decisión de abandonar la relación exclusiva del concepto de innovación (de producto o procesos), con los cambios tecnológicos; esto es: se incorporan las innovaciones en servicios. 
"The identification of product and process innovation with technological change was abandoned in order to include service innovations that significantly improved user experiences without necessarily having a technological component" (OCDE - Manual de Oslo, 2018 en referencia a lo comenzado en su versión del 2005).

"La identificación de innovación de procesos y productos con el cambio tecnológico, fue abandonada con vistas a incluir innovaciones en servicios que mejoran significativamentela experiencia del usuario sin necesariamente contener un componente tecnológico" (Traducción de los autores)

Asimismo, en la cuarta edición, amplía la dimensión de medición de la innovación con nuevas incorporaciones:

"This fourth edition of the Oslo Manual takes account of major trends such as, the pervasive role of global value chains; the emergence of new information technologies and how they influence new business models" (Manual de Oslo, 2018).

"Esta cuarta edición del Manual de Oslo, toma en cuenta tendencias mayores como, la penetración del rol de las cadenas globales de valor; la aparición de las tecnologías de la información y como estas influencian a los nuevos modelos de negocios" (traducción del autor).

En el cuaderno de trabajo Innovation Concepts And Typology - An Evolutionary Discussion (M. Kotsemir M, Abroskin A, Dirk M, 2013); los autores, desarrollan la evolución del concepto de innovación, colocando el énfasis en la recopilación de los diversos modelos de innovación que se fueron desarrollando a través de los años por diversos autores; a efectos de construir una suerte de tipología de dichos modelos.

La tabla 1 resume los modelos y corrientes mencionados en el estudio, rescatando los que presentan a la innovación desde dimensiones que no necesariamente se asocian a la tecnología, (la tabla menciona aquellos modelos que han adquirido mayor relevancia en el tiempo, y perduran hasta la actualidad).

Tabla 1 - Modelos y filosofías de Innovación

\begin{tabular}{|l|l|c|}
\hline \multicolumn{1}{|c|}{ Tipo de innovación } & \multicolumn{1}{|c|}{ Autores Referentes / Creadores } & Año de origen \\
\hline Nicho & Abernathy and Clark (1985). & 1985 \\
\hline Arquitectural / Modular & Henderson and Clark & 1990 \\
\hline Nuevo Modelo de Negocios & $\begin{array}{l}\text { Autores varios (Como concepto tiene su origen } \\
\text { en el artículo de Peter Drucker Teoría del } \\
\text { Negocio, publicado en la Harvard Bussines } \\
\text { Review en 1994). Aunque Drucker nunca utilizó } \\
\text { el término modelo. }\end{array}$ & 1995 \\
\hline $\begin{array}{l}\text { Innovación en Valor (Red / } \\
\text { Blue Ocean) }\end{array}$ & Mauborgne R, Kim W. & 2005 \\
\hline & Moore G. (2005) & 2005 \\
\hline Innovación Orgánica & Slywotzky A (1996), Moore G. (2005) & \\
\hline Migración de Valor & Radjou N,Jaideep Prabhu J. & 2005 \\
Innovación Frugal & & \\
\hline
\end{tabular}

Fuente: AUTOR. 
"La idea de quitar la carrocería de un camión y cargarlo en un barco, no involucra demasiada tecnología. Esa innovación, el contenedor, no nació de un avance tecnológico, sino de una percepción nueva en cuanto a lo que podía ser un barco que cargara contenedores, y no la carga directa". (Drucker $\mathrm{P}, 1986)$.

En el libro La innovación y el empresario innovador, Peter Drucker enumera y desarrolla siete areas o fuentes de potencial innovador, dentro de las cuales solo una de ellas refiere a la ciencia y tecnología.

Obsérvese que las áreas o fuentes de valor mencionadas por Drucker, se proponen como áreas o fuentes de búsqueda que permiten hallar innovaciones. En este caso, la tecnología está incluida en el área siete (Nuevos conocimientos y nuevas ciencias) como fuente de búsqueda. Un tratamiento mayor se realizará en el marco teórico.

En el artículo Innovation: Mapping the Winds of Creative Destruction (Aberthany W, Clark K, 1986), los autores mencionan:

"Novedad y su conexión con el avance científico pueden tener poco que ver con una significativa innovación competitiva. El ingreso de la Timex Corporation en la industria de los relojes provee de un útil ejemplo. Su éxito en el mercado estuvo sustentado en el refinamiento de una vieja tecnología...". (Aberthany W, Clark K, 1986, traducción del autor).

En dicho artículo, los autores exponen un modelo de matrices en el que quedan definidos cuatro formatos de innovación: Innovación de Nicho, Innovación de Arquitectura, Innovación Revolucionaria, Innovación Regular, en la que solo una de las dimensiones del modelo incorpora cambios el la tecnología.

Asimismo, en dicho artículo, los autores explican que el concepto de destrucción creativa surgido de Joseph Schumpeter a partir de las innovaciones llamadas radicales (nuevas tecnologías que irrumpen con profunda afectación en los ámbitos económicos y sociales), es solo una de las formas de innovación, y que se produce en raras ocasiones. Sobre estas innovaciones mencionan:

"Es un estereotipo de un ideal que es rara vez encontrado en la práctica y engañoso" (W, Clark K, 1986, traducción del autor)

Es por supuesto innegable que, en numerosos casos la tecnología sí se expone como el factor clave de una innovación; fundamentalmente cuando se trata de tecnologías nuevas o recientes y/o las denominadas como alta tecnología. Lo que nos preguntamos aquí es si toda innovación está sustentada exclusivamente en el factor tecnológico como elemento clave; y aún en los casos de las innovaciones que incorporan altas tecnologías, también cabría la misma pregunta.

Nuestro propósito en el presente estudio, ha sido tomar muestras de innovaciones, y observar si es posible discriminar en cada una de ellas el aspecto no tecnológico (o componente no sustentado en la tecnología de punta); para luego a partir de estos, proceder a evaluar si dicho componente puede concebirse como un factor aplicativo u orientativo respecto a otras areas, productos, negocios, modelos de negocios, ETC. A dichos componentes a los hemos denominado: factores aplicativos u orientativos.

Para echar luz sobre el tema brindaremos dos ejemplos: La empresa LG ha lanzado un modelo de pantalla de TV que puede enrollarse, para logrargo utiliza una nueva tecnología denominada OLED (Organic LED). Por otra parte, la empresa la empresa VolkerWessels se dedica a un proyecto de construcción de carreteras de plástico que pueden instalarse como bloques 
modulares. Para la realización del proyecto, existen varios aspectos tecnológicos implicados; sin embargo el factor novedoso y útil del proyecto es el aspecto de construcción modular, sumado a la sostenibilidad del uso de plástico; dicho aspecto es un factor clave que no se sustenta en la tecnología de punta.

Puede identificarse en este ejemplo, que la innovación tiene un sustento completo en una tecnología de punta.

Desde ya que el proyecto de Rotterdam involucra aspectos tecnológicos, sin embargo ese componente de construcción modular, es un factor clave de dichas innovaciones, que no está relacionado con la utilización de la tecnología de punta, sino con una forma diferente de pensar la construcción.

Cabe destacar que el foco de la presente investigación es la innovación como resultado $u$ output (un producto, un nuevo negocio o formato de negocio, un abordaje distinto y util, o un nuevo proceso) y no la innovación en otras dimensiones.

\subsection{Objetivos}

En función a lo expresado en la introducción, los objetivos de la presente investigación son los siguientes:

1. Verificar la existencia de factores clave no tecnológicos, en las innovaciones no sustentadas en Alta Tecnología.

2. Desarrollar agrupamientos de las innovaciones registradas, en función a los factores clave mencionados, compartidos entre estas.

3. Relacionar cada agrupamiento con los modelos, filosofías y perspectivas teóricas de la literatura y corrientes de autores.

4. Sentar las bases para la construcción futura de un modelo de guía y orientación, que en función de los factores clave mencionados; pueda ser utilizado como herramienta para acotar el espacio de búsqueda al momento de generar potenciales innovaciones.

5. Desarrollar un método analítico o de descomposición para el análisis de los factores no tecnológicos de una innovación.

\subsection{Justificación}

La existencia misma de factores subyacentes a las innovaciones no sustentadas en tecnología de punta, y cuya aplicación sea susceptible de ser replicada (dando lugar al desarrollo de otras innovaciones); abre sólidas posibilidades en diversos ámbitos:

En primer lugar y desde la perspectiva empresarial y del emprendedorismo, la posibilidad de contar con una metodología que permita la obtención sistemática de potenciales innovaciones, otorga ventajas competitivas per se; dando lugar a entidades (empresas, regiones, emprendedores, etc.) que contarían con un modelo consistente de desarrollo innovador, el cual no estará únicamente sustentado en la posibilidad de contar con el acceso a la alta tecnología.

El mismo razonamiento puede aplicarse a las políticas económicas regionales o sectoriales, ya que de existir principios subyacentes en las innovaciones que hoy prosperan en los mercados, sin que su desarrollo haya estado originado en la generación de alta tecnología; significará en muchos casos que dichos principios obedecen a nuevas formas de combinación o guías de búsqueda de potenciales oportunidades, como así también a nuevos modelos de negocios que, 
aplicados a diversas regiones o sectores permitirían potenciar la competitividad de los mismos a un costo menor que si se requiriese contar con tecnologías fuera del alcance de inversión de los mismos.

Lo anterior es por supuesto, sin detrimento de ser concientes del grado de validez que la inversión de I+D ofrece en referencia al crecimiento de la riqueza de una nación, región, empresa o sector. Por último, son escasos los trabajos empíricos acerca de las innovaciones que no se sustentan en tecnología. No hemos encontrado en la literatura, trabajos que a partir de la recopilación y descomposición de casos, analicen las innovaciones no sustentadas en tecnología a efectos de encontrar similitudes y factores en común.

\section{FUNDAMENTACIÓN TEÓRICA}

\subsection{Economía e Innovación}

Sea tal vez el economista austríaco Joseph Schumpeter con su visión de la llamada destrucción creativa (Schumpeter J, 1942) el primer exponente de la investigación de las innovaciones como motor de crecimiento y desarrollo de las economías, aunque como indica Carlota Perez (Perez C, 2010), su enfoque se dirigió hacia las características y virtudes de aquel que el mismo Schumpeter dio a llamar el empresario innovador (Schumpeter J, 1942) y la relación entre los procesos de innovación y el desarrollo capitalista.

Desde las bases de los trabajos de Schumpeter, surgiría un nuevo conjunto de adeptos, quienes con el tilde de Neoschumpeterianos, iniciarían un estudio sistemático y organizado de los procesos innovadores, ya no haciendo hincapié en el empresario innovador como Schumpeter, sino en el estudio de las innovaciones como un proceso evolutivo que deja registro a través de un ciclo de vida que inicia en innovaciones denominadas radicales, hasta que la misma innovación llega a una etapa de declive, dando lugar a una nueva revolución innovadora que surge asimismo de una nueva oleada de innovaciones, también de carácter radical.

Dentro de estos seguidores, se alza Christopher Freeman como uno de los más relevantes, quien a través de sus trabajos académicos daría fuerza al resurgimiento de las ideas de Schumpeter pero desde la óptica evolutiva mencionada.

Sin embargo, el trabajo de los Neoschumpeterianos tiene su enfoque en la mirada de la innovación industrial, técnica y tecnológica: su evolución, sus patrones regulares e irregulares de aparición, las características de los sistemas nacionales de innovación que permiten o no el desarrollo innovador. Su visión se fundamenta en una tecno-evolución y la detección de los ciclos de la misma, como así también cual es el origen de los proceso de innovación en la industria (Freeman C, 1998).

Todo trabajo posterior a Freeman, hasta llegar a la actualidad con una exponente como Carlota Pérez, tratan a la innovación desde la misma perspectiva, analizando sus fuentes de desarrollo, sus características evolutivas y su impacto económico social, y más recientemente financiero (Perez C., 2002).

\subsection{La innovación desde la perspectiva de la capacidad sistemática de inventar}

El estudio de la innovación caló también en el ámbito de la ingeniería, fundamentalmente (y más allá de la tecnología como base de nuevos productos y procesos) como el estudio de la inventiva y la capacidad de generar soluciones a problemas, e idear nuevos desarrollos. 
Desde este ámbito, el trabajo de G. Altshuller destaca, como así también su historia. Altshuller fue un ingeniero que trabajó en el departamento de patentes de la pasada Unión de Repúblicas Socialistas Soviéticas en la época de Stalin.

Su labor en el departamento de patentes, le permitió registrar y analizar las invenciones técnicas de miles de casos, derivando luego de varios años de investigación y de análisis, una serie de patrones que seguían a las invenciones de tipo técnicas.

Altshuller desarrolló entonces toda una metodología para la "inventiva", en la que destacó el desarrollo de una Matriz de Contradicciones técnicas de ingeniería. La matriz consiste en una tabla de 39 parámetros de ingeniería que en la práctica de la actividad pueden presentarse como contradicciones, esto significa que todo problema de ingeniería puede presentarse como un conflicto entre un par de parámetros, a modo de ejemplo: incrementar la velocidad máxima de un vehículo, se utiliza un motor más potente y, por consiguiente más pesado, por ende el parámetro velocidad presenta una contradicción con el parámetro peso. Su primer trabajo data del año 1961, con la publicación de su libro Cómo aprender a inventar.

El trabajo desarrollado por Altschuller se estudia en las cátedras de ingeniería de diversas casas de altos estudios, como así también su aplicación se ha realizado en diversas empresas de primer orden como Ford, Alcatel, Bic, Xerox y Christian Dior entre otras. Incluso es mencionado y/o referido en numerosos artículos de investigación y divulgación: Finding your innovation sweet spot (Goldenberg J., 2003) de la Harvard Business Review es un ejemplo de ello.

El trabajo de Altshuller es uno de los pocos y reconocidos desarrollos fundamentados desde la investigación, que expone un cuerpo metodológico estructurado para la generación de nuevas ideas innovadoras y la solución de problemas de su ámbito disciplinar (La ingeniería). Su mención aquí se debe a que es uno de los trabajos que mayor analogía presenta con respecto a la presente investigación: la de encontrar patrones sistemáticos para el desarrollo de innovaciones, aunque en un ámbito distinto de actuación.

\subsection{El ámbito empresarial}

Ya hacia el año 1985 el prominente académico Peter Drucker, en su obra La innovación y el empresario innovador (Drucker $\mathrm{P}, 1985$ ), comienza a introducir la innovación como un área de análisis dentro de su competencia: el Management y/o el estudio de la disciplina de gerenciar, en resumen: la gestión de la empresa.

Allí mismo presenta siete áreas de exploración de valor u oportunidades de innovación para el empresario, siendo el académico más prominente de su época en proponer el proceso de desarrollo de innovaciones como una búsqueda sistemática. Fue uno de los primeros acercamientos a una visión del proceso de desarrollo de innovaciones en el ámbito empresarial con un método sistemático, esto es: un método que expone siete focos en donde comenzar a descubrir oportunidades de innovación.

\subsection{La actualidad: Innovación en valor y modelos de negocios}

La primera aparición de profundo impacto posterior a Drucker acerca del estudio y construcción de una metodología enfocada en la generación de innovación de valor en la empresa, aparecería recién en el año 2005 (Chan Kim w., Mauborgne R, 2005) en su libro La estrategia del Océano Azul.

El libro tiene su origen en un artículo de investigación que los autores publicaron en la Harvard Business Review en Julio de 2004: Value Innovation: The Strategic Logic of High Growth. 
Es tal vez la primera incursión en la sistematización de un proceso de desarrollo de innovaciones en valor. La metodología de los autores se sustenta en el estudio y análisis de casos de empresas innovadoras de diversas industrias en los últimos cien años. El proceso los llevó al desarrollo de una serie de herramientas y métodos de análisis para encontrar lo que ellos denominan innovación en valor. Es el método sistemático (dirigido a la empresa y su aplicación) que se presenta como uno de los de mayor sustento académico e investigativo hasta la fecha.

Cabe destacar que si bien existen múltiples publicaciones del tema, muchas de ellas surgen de artículos de divulgación de respetados autores, más que de investigaciones. La visión de los autores de La estrategia del Océano Azul supone la construcción de un modelo de exploración de oportunidades que haga irrelevante la necesidad de competir en una industria. La metodología presenta herramientas analíticas para lograr construir ventajas competitivas diferenciales, logrando formatos de negocios que sean innovadores frente a la industria donde la empresa en cuestión tiene su actuación.

Al igual que Drucker, aunque con mayor rigor metodológico y robustez que aquel, proponen seis vías de exploración de valor en donde enfocarse para lograr visualizar oportunidades de innovación, y un método denominado cuadro de valor, para realizar comparaciones de valor con la industria, en las que proponen seis áreas de exploración de innovación en valor.

Asimismo, presenta una herramienta denominada cuadro estratégico de valor, en donde se presenta el desempeño de la industria en función a una serie de factores comunes, y se compara la propuesta de valor de la empresa respecto a la industria donde compite, en función a cambios propuestos sobre dichos factores; agregando, quitando, aumentando o reduciendo los mismos factores u otros nuevos.

\subsection{Los modelos de negocios}

Continuando con el ámbito de la estrategia empresarial, un concepto que se relaciona con la innovación es el de Modelo de Negocios. Introducing a better business model into an existing market is the definition of a disruptive innovation (Ovans A., 2015). El concepto de Modelo de negocios fue utilizado por primera vez en el libro The New, New thing del periodista económico y escritor estadounidense Michael Lewis en el año 1999. El libro de Lewis enfoca su atención en las empresas de Sillicon Valley y fundamentalmente las empresas punto com.

El mismo autor menciona el término modelo de negocios, asignándole la característica de una forma de arte que es reconocida cuando se está frente a ella pero de difícil definición (Lewis M., 1999). Asimismo, en el mismo libro menciona que All it really meant was how you planned to make money (Lo que en realidad significó fue como se planeaba generar dinero), haciendo alusión a que en esencia, ese era el punto de las compañías en internet con sus nuevos modelos de negocio.

Es importante destacar que si bien el concepto nace en dicha publicación, lo que este implica data de varias décadas anteriores, aunque no con esas palabras. La definición de modelo de negocios posee varias propuestas, muchas de ellas generalmente aceptadas, aunque partiendo de diversas perspectivas; mientras algunas de ellas parten del objetivo que se asume como final: "...la forma de planear como hacer dinero" (Lewis M., 1999), otras se enfocan en el valor: "Es la forma en que la empresa crea y entrega valor a sus clientes" (Osterwalder, 2005). Asimismo, es frecuentemente utilizada la definición de Peter Drucker (aunque este nunca utilizó el término "modelo") que indica que es un conjunto de supuestos (Drucker P., 1994) que guían el diseño 
estructural de determinado negocio para generar beneficios hoy y en el futuro (su visión, infraestructura, su relación con los clientes, sus competencias centrales, etc.).

La relación entre modelo de negocios e innovación, surge del hecho que un nuevo modelo de negocios implica necesariamente una perspectiva diferente; se construye sobre supuestos y diseños de negocios que, mediante abordajes distintos a los de la industria en donde se actúa y compite, intentan generar valor sustancialmente diferencial.

\subsection{Desarrollo conceptual de los modelos de innovación}

Hemos expuesto hasta el momento las grandes líneas más relevantes en referencia al estudio de la innovación, sin embargo a través de los años y desde que el management irrumpió como disciplina en la escena empresarial; existieron otros modelos y filosofías de innovación que orientan la mirada de la innovación en la dirección del estudio, esto es: la innovación no sustentada en tecnología de punta. Describiremos brevemente algunos de estos modelos o líneas de pensamiento.

\subsubsection{El modelo de Innovación de Henderson and Clark}

En el año 1985, Rebecca Henderson y Kim Clark desarrollaron un modelo de innovación matricial en el quedan definidos cuatro tipos de innovaciones (figura 1)

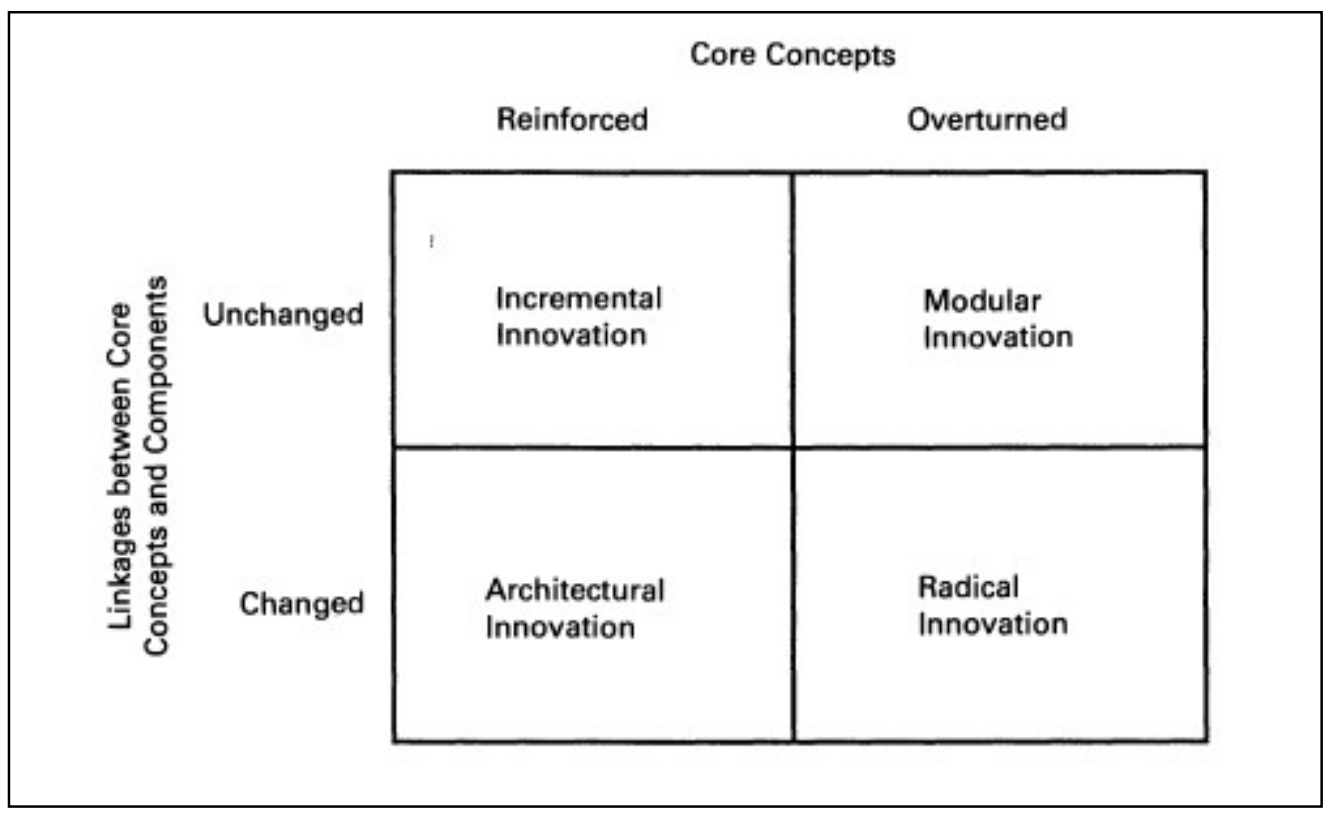

Fuente: Henderson M, Clark K (1990)

Figura 1 - Modelo de Henderson and Clark

Mientras los conceptos de innovación incremental (Incremental Innovation) e innovación radical (Radical Innovation) son los mismos que los desarrollados por Joseph Schumpeter (Shcumpeter, 1944); los autores presentan dos nuevas dimensiones: Innovaciones de Arquitectura e Innovaciones Modulares. En este modelo, una innovación es de arquitectura cuando en un producto, pueden intercambiarse selectivamente uno o varios de sus modulos, sin afectar al resto de los módulos; y es modular, cuando lo que se modifican son las piezas dentro de un módulo, pero no se modifica la relación entre los módulos del artefacto. 


\subsection{2 Áreas de de búsqueda de innovacion de Peter Drucker}

Como hemos mencionado con anterioridad, en su libro La innovación y el empresario innovador (Drucker $P, 1985$ ). En la tabla 2 se presenta un cuadro en el que se menciona cada una de estas áreas, con una suscinta descripción de cada una.

Tabla 2 - Áreas de Innovación - Drucker P.

\begin{tabular}{|l|}
\hline \multicolumn{1}{|c|}{ Fuente de Innovación } \\
\hline Observar los eventos inesperados \\
\hline Analizar lo incongruente \\
\hline Detectar el proceso faltante \\
\hline Observar la estructura de mercado y de la industria \\
\hline Medir los cambios demográficos y las tendencias. \\
\hline Observar los cambios en la percepción, modalidad y significado (El vaso medio lleno) \\
\hline Incorporar nuevos conocimientos, tanto científicos como no científicos. \\
Fuente: AUTOR
\end{tabular}

\subsubsection{El modelo de innovación en valor de Mauborgne y Kim}

La innovación en valor es un concepto desarrollado por los autores en el libro La estrategia del Océano Azul (Mauborgne R, Kim W, 2005). El modelo se sustenta en el hecho que en cada industria, se compite sobre diversos parámetros o dimeniones de valor (la entrega, el precio, el segmento, el tipo de comunicación, las promociones, el estilo, entre otras dimensiones, dependiendo de la industria en cuestión); por lo tanto, para que una empresa pueda generar una innovación en valor, debe analizar modificaciones en el peso de algunos de esos parámetros, y esto se logra agregando nuevos parámetros, eliminando parámetros que suman costos pero reducen valor, e incrementando y/o reduciendo el valor invertido en alguno de los parámetros o dimensiones de la industria.

A efectos de auxiliar en el análisis, los autores proponen seis vias de exploración de valor (tabla 3).

Tabla 3 - Seis Vías de Valor / La Estrategia del Océano Azul

\begin{tabular}{|l|}
\hline \multicolumn{1}{|c|}{ Vías de exploración de valor } \\
\hline Exploración de industrias alternativas. \\
\hline Exploración de los grupos estratégicos. \\
\hline Exploración de la cadena de compradores. \\
\hline Exploración de las ofertas complementarias de productos y servicios. \\
\hline Exploración del atractivo funcional o emocional de los compradores. \\
\hline Exploración de la dimensión del tiempo. \\
\hline
\end{tabular}
Fuente: AUTOR

\subsubsection{La filosofía de la Innovación Frugal de Radjou $N$ y Prabhu J}


La filosofía propuesta por los autores, está implicada en el "hacer más con menos", en el libro Frugal Innovation: How to Do More with Less (N Radjou, J Prabhu, 2015), los autores presentan una serie de principios que orientan a la generación de innovaciones a partir de esta filosofía. La innovación frugal busca innovar manteniendo solo las funcionalidades, materiales y procesos esenciales del producto; y evitando la incorporación de dimensiones decorativas o accesorias; con objeto de obtener productos viables para mercados masivos de menores recursos.

2.6.5 El concepto de innovación Inversa de Dartmouth Vijay Govindarajan, Chris Trimble y Jeffrey $R$ (2009)

De acuerdo a los autores, los productos desarrollados para mercados masivos de bajos recursos y naciones en desarrollo; pueden luego comercializarse en naciones desarrolladas, como innovaciones de bajo costo ("low cost" innovations).

\subsection{Innovación e innovación tecnológica}

\subsubsection{Medición de la tecnología}

El Manual de Frascati, perteneciente a la familia de documentos de la OCDE, ofrece las guías y orientaciones para la recopilación, reportes y uso de los datos referidos a investigación y desarrollo. Hasta el año 2003 un método para discriminar los niveles de tecnología era la intensidad en Investigación y Desarrollo Experimental (I+D); y era utilizado solo para las industrias manufactureras (Isic Rev. 3 Technology Intensity Definition - OCDE, 2011). Este método segmentaba industrias en cuatro niveles (Alta, Medio alta, Media y Baja tecnología). Esta medición se realiza en función a los gastos en Investigación y Desarrollo de dicho sector, versus el producto bruto interno.

A partir del 2010, y explicitado en el cuaderno de trabajo OECD Taxonomy of Economic Activities Based on R\&D Intensity (OCDE, 2016), se propone una nueva taxonomía de las industrias, adoptando solamente la clasificación en niveles de intensidad en $I+D$ y abandonando el concepto de intensidad tecnológica (OECD Taxonomy of Economic Activities Based on R\&D Intensity, 2016). La OCDE continúa con las mediciones de ciencia y tecnología, sin embargo fue descartada la clasificación asociada a la intensidad tecnológica.

\subsubsection{Medición de la innovación}

La OCDE mide también a la innovación (mediante las directrices de su Manual de OSLO). En los últimos años, las diversas ediciones del Manual de Oslo, fueron adicionando suscesivas modificaciones a través de la consideración de diversas dimensiones del fenómeno de la innovación.

La innovación se mide a través de encuestas a empresas. A partir de su edición de 2018, las encuestas se enfocan en dos mediciones particulares: las actividades e inversiones que se desarrollan en función de generar una innovación, y las innovaciones como resultado de dichas actividades: subject approach and object approach (OCDE - Manual de Oslo, 2018).

"Una innovación es un nuevo o mejorado producto o proceso (o combinación de estos) que difiere significativamente de los productos o procesos previos desarrollados por la unidad productiva y que se han colocado a disposición del usuario potencial (producto) o incorporados en el uso por la unidad productiva (proceso)" (Manual de Oslo, 2018, traducción del autor).

Mientras que la primera y segunda edición del manual de 1992 y 1997, se medía exclusivamente la innovación en la industria de manufacturas, su tercera edición en 1997 incorporó a las industrias de servicios. 
"La primera y segunda ediciones limitaron el concepto de innovación, a nuevos o significativamente mejorados productos y procesos 'tecnológicos' " (OCDE - Manual de Oslo, 2018, traducción del autor).

En su tercera edición (2005) se expande la perspectiva de la medición, dando mayor énfasis al rol de los procesos de innovación realizados en colaboración con otras empresas, en lugar de considerar solo la dimensión de los cambios de una empresa en particular. Asimismo, se reconoce la necesidad de incorporar en la medición a los sectores que tradicionalmente poseen menor intensidad tecnológica. Asimismo se decidió abandonar la identificación exclusiva de productos y procesos de innovación, con el cambio tecnológico; en vistas de incluir innovaciones en servicios que mejoran sustancialmente la experiencia de los usuarios, sin que necesariamente incorporen componentes tecnológicos.

A partir de su versión de 2018, la OCDE comienza a discriminar entre actividades de innovación (como proceso - subject approach) y la innovación como resultado (outcome, object approach). En las figuras 2 y 3 , podemos observar un comparativo de naciones respecto a su intensidad en I+D en industrias manufactureras, que se utilizaba anteriormente como medida de la intensidad tecnológica (figura 2); y la medición de empresas innovadoras para el año 2016 (figura 3); ambos cuadros fueron elaborados mediante la herramienta online Scoreboard Monitor del sitio online de la OCDE.

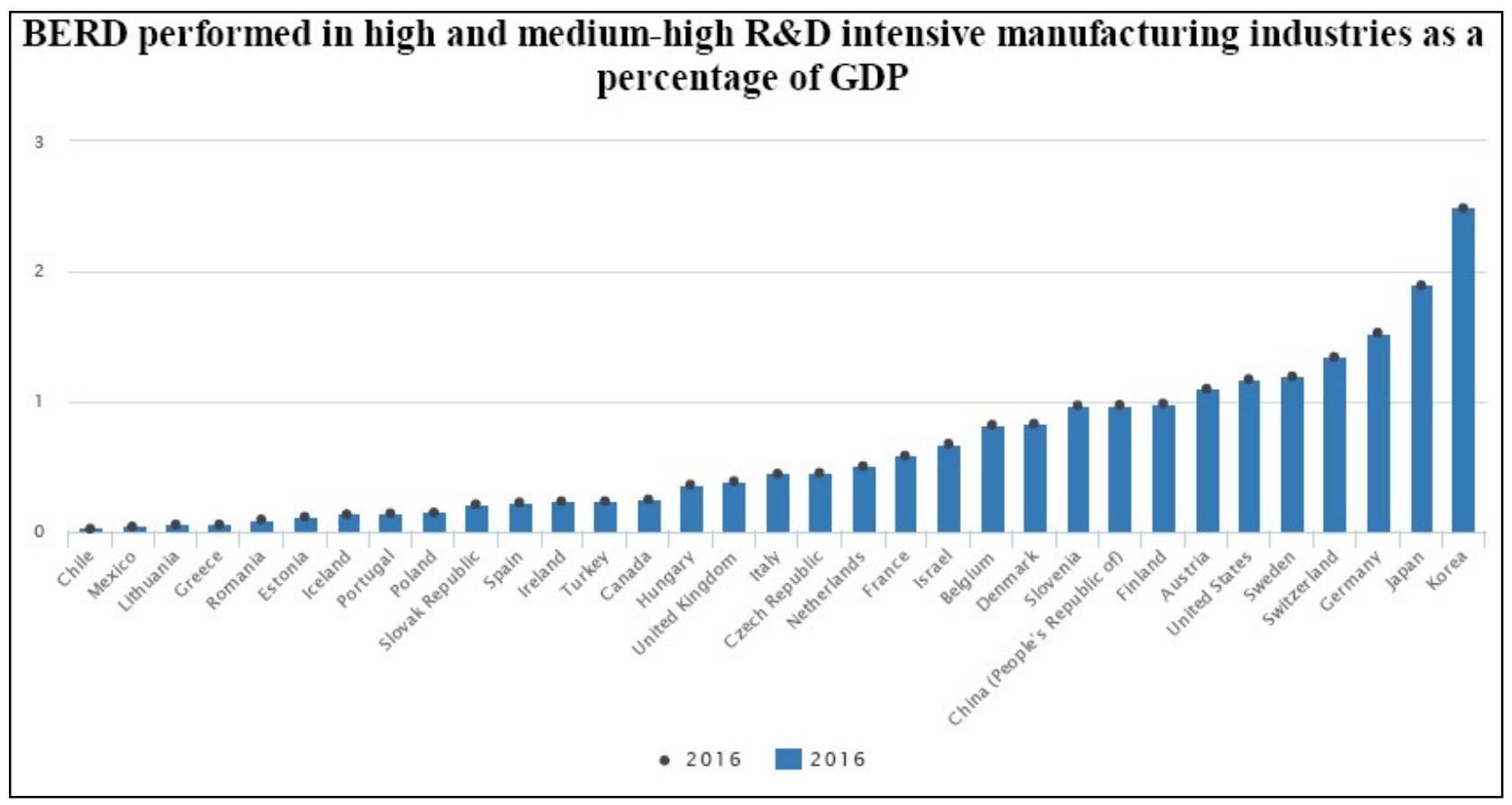

Fuente: OECD ScoreBoard Monitor

Figura 2 - Intensidad en I+D versus PBI 


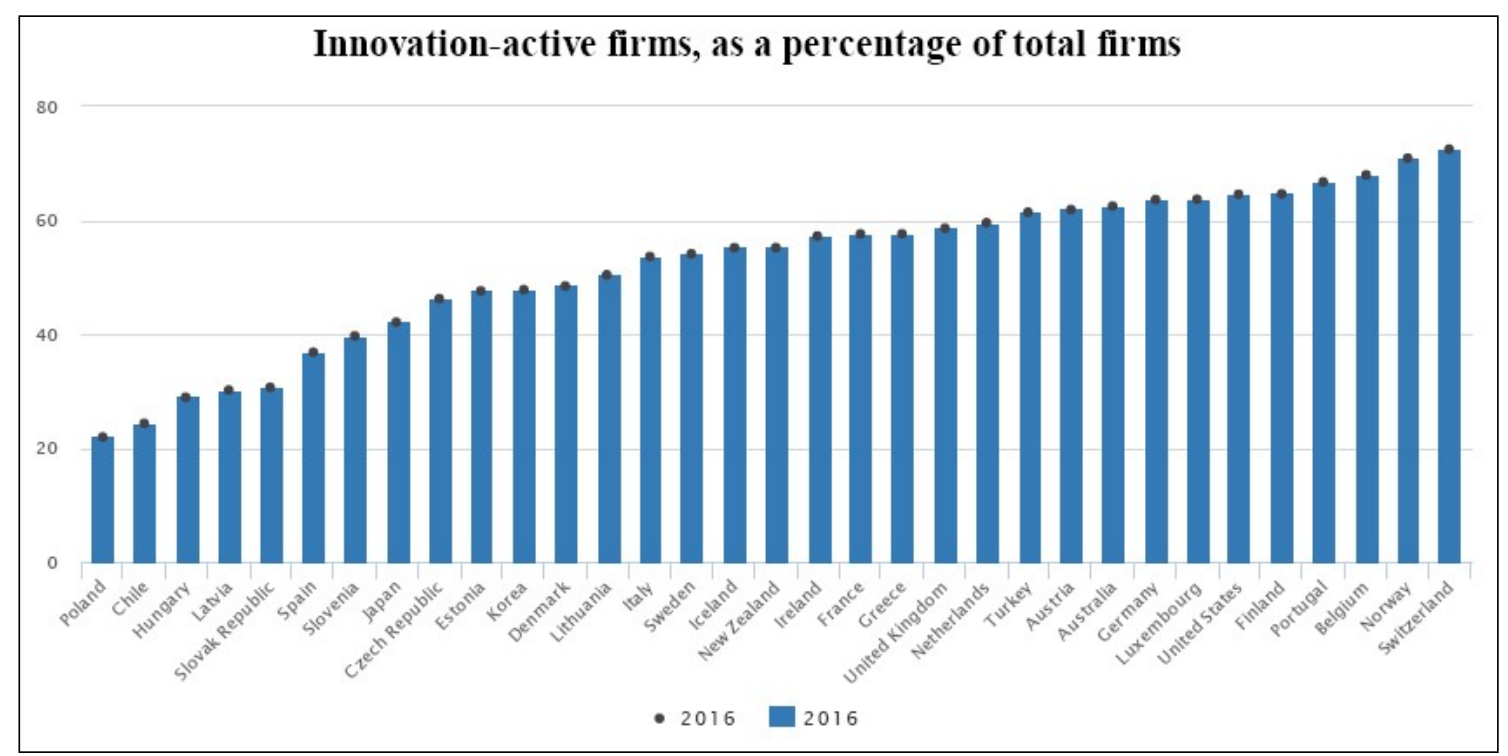

Fuente: OECD ScoreBoard Monitor

Figura 3 - Empresas con actividad Innovadora

En ambas imágenes (tomados al año 2016) puede observarse que existen diferencias en el orden en que los países aparecen; a modo de ejemplo, Israel fue en 2016 el país que mayor proporción de gastos de I+D en su industria manufacturera sobre el PBI ha realizado; sin embargo no se encuentra entre las veinte primeras posiciones de las naciones en lo referente a porcentaje de empresas con actividad innovadora, sobre el total.

De la misma forma, Noruega, en segundo lugar detrás de Suecia en tasa de empresas con actividad innovadora (sobre el total de empresas), no se encuentra en ninguno de los puestos de I ranking de intensidad de I+D en manufacturas; Suecia se encuentra en el cuarto puesto de dicho ranking. Japón, segunda en el ranking de intensidad en I+D en la industria de manufactiras, no alcanza los primeros quice puestos dentro del ranking de firmas con actividad innovadora.

Es de notar que existe un progresivo trayecto de la OCDE, hacia una medición de la innovación en dimensiones que no implican necesariamente los aspectos de $I+D$, y al mismo tiempo, abandonando la asociación de I+D con clasificaciones de intensidad tecnológica; La diferencia entre las posiciones de las naciones en ambos cuadros, evidencian que la innovación es un fenomeno que excede los bordes de la incorporación de $I+D$, como así también una discriminación respecto a la tecnología como factor clave.

\subsection{El problema de innovar y el concepto de espacio de búsqueda o soluciones}

El concepto de espacio de búsqueda, o acotación del espacio de soluciones; es ampliamente utilizado en problemas complejos matemáticos combinatorios; fundamentalmente cuando no existen modelos deterministas para hallar una solución, dado que en estos casos lo que se busca es alguna solución heurística que acote dicho espacio de búsqueda de soluciones.

Extrapolando el concepto a nuestro estudio, espacio de búsqueda, se refiere a cuan vasto es el conjunto de áreas, fuentes y dimensiones que deben ser exploradas por una unidad productiva (empresa, emprendor, institución, ETC.), al momento de iniciar un proceso de generación de potenciales innovaciones. Por lo tanto, los factores aplicativos u orientativos que se 
han hallado, tienen como finalidad un acotamiento de dicho espacio de búsqueda, al momento de iniciar un proceso de generación de innovaciones.

\section{METODOLOGÍA}

Podemos enmarcar la presente investigación como Exploratoria - Descriptiva. La investigación nos propone asimismo, incorporar la utilización del método analítico a efectos de descomponer el objeto de estudio (las innovaciones), y estar en condiciones de realizar la detección y clasificación de sus principios heurísticos clave.

\subsection{Recolección de datos}

Dado el volumen y diversidad de sitios, organismos, revistas de interés general y revistas digitalizadas del ámbito empresarial, revistas científicas y de universidades; que presentan ejemplos, casos y novedades de productos y negocios; siendo que al mismo tiempo ninguna de estas por si mismas ofrece la cantidad o representatividad necesarias para realizar un estudio; las fuentes se presentan con una gran diversidad y salvos casos de excepción, se vuelcan a continuación los tipos de fuentes.

Sin embargo, enunicaremos los descriptores utilizados para las búsquedas, los que luego nos llevan a dichas diversas fuentes. Asimismo, en la tabla de casos recopilados que figura en el anexo, se hace referencia con link para evidenciar la fuente de cada muestra.

La recolección de datos se ha realizado mediante:

- Revistas dedicadas al ámbito de nuevos productos, negocios; del ámbito empresarial.

- Bibliografía específica de tendencias.

- Casos de la OCDE (Organización para la cooperación y el desarrollo económicos).

- Harvard Bussiness Review.

- Indiegogo (sitio de start ups).

- Casos de estudio - publicados a través de Elsevier.

- The Monopolitan - revista de tendencias.

\subsection{Descriptores de búsqueda y requisitos para la recopilación de las muestras}

En las búsquedas, no necesariamente las innovaciones aparecen mencionadas con dicho vocablo o término; por ende se requirió utilizar diversos descriptores de búsqueda provenienes de términos asociados al de innovación y no solo la palabra innovación. En función a lo mencionado, los términos utilizados como descriptores fueron:

Innovación, Innovación sin tecnología, innovación sin alta tecnología, productos innovadores, nuevos productos, productos novedosos, productos originales, productos ingeniosos, servicios innovadores, servicios originales, nuevos servicios, nuevos modelos de negocios, nuevos formatos de negocios, nuevos negocios, negocios originales, negocios novedosos, negocios innovadores. emprendimientos originales, emprendimientos innovadores, emprendimientos novedosos, emprendimientos ingeniosos, soluciones innovadoras, soluciones novedosas, soluciones originales.

Las condiciones de las innovaciones a recopilar son las siguientes:

- Se tomarán los casos producidos en los últimos 20 años.

- Solo se considerarán aquellas innovaciones cuyo factor de utilidad y novedad, no estén sustentadas en la Alta Tecnología (independientemente que la incorporen).

- Solo se considerarán las innovaciones que, sin importar el momento de su introducción, aún se encuentren en el mercado. 
- Se refieran a nuevos o sustancialmente modificados bienes, servicios y/o novedosos formatos de negocios.

- El valor que ofrezcan dichas innovaciones esté referido al ámbito funcional o estéticofuncional; se descartan las innovaciones exclusivamente estéticas; como así también se descartan las innovaciones cuyo factor clave implique cambios en aspectos de orden psicológico, cognitivo, emotivo ó simbólicos.

- Se descarta el aspecto ingenieril de la innovación. No está dentro del alcance del estudio el mecanismo por el cual se llega a construcción del objeto (en el caso de bienes tangibles), salvo cuando ese mecanismo este sustentado exclusivamente en alta tecnología, caso en que queda excludido.

\subsection{Muestreo}

Se utiliza un tipo de muestreo aleatorio por estratos. Los estratos se realizarán considerando la categorización de los países en función a su desarrollo, para ello se utilizará el IDH (Índice de Desarrollo Humano) que elabora el PNUD (Programa de las Naciones Unidas para el Desarrollo). Este índice divide a los países en cuatro grandes grupos.

Dentro de cada estrato, la proporcionalidad de representación se realizará en función a la sumatoria de la cantidad de patentes registradas por los países de cada grupo; dato a obtener del índice de desarrollo innovador de la OMPI. El tamaño de la muestra se analizará al momento de iniciar el estudio, aunque en principio se verifica una población infinita. Luego se aplicarán las proporciones mencionadas.

\subsection{Variables a analizar}

Dentro de cada innovación se procederá a detectar el/los principio/s heurístico/s clave que otorgan a la misma las dos características esenciales de las innovaciones: novedad y valor (o utilidad).

\subsection{Procedimiento de discriminación, análisis y agrupamiento de las muestras}

El método analítico juega aquí un papel preponderante, dado que lo que aspiramos a obtener es la detección de las características específicas que convierten el abordaje en innovador, sin que dicha característica esté sustentada en la tecnología de punta; aún cuando ducha muestra pueda incorporar dicho nivel de tecnología. Luego de la recopilación de la muestra, el proceso procedió de la siguiente forma:

1. Se verificó la existencia o no de tecnología de punta.

2. Se analizó la muestra, discriminando si existe un factor que le otorga el carácter de novedosa, al margen de la tecnología de punta que pueda incorporar.

3. Se procedió a nominar y registrar dicho factor.

4. A medida que avanzó la recopilación y análisis, se procedió a detectar si las nuevas muestras coinciden con factores ya nominados.

5. Se procedió a agrupar todas las muestras en dichos factores.

6. En caso que una misma muestra responda a más de un factor simultáneamente, dicha muestra se nomina en función a ambos factores.

7. Se procedió a analizar cada factor en función a la literatura sobre el tópico; los modelos y perspectivas de las corrientes teóricas.

8. Por último, se relacionó cada grupo de factores, con la corriente, modelo o teoría pertinente. 
Hasta el momento se han analizado 284 muestras, que representan 342 casos, esto es así debido a que algunas de las muestras pueden clasificarse en más de un principio o factor simultaneamente.

\section{RESULTADOS Y DISCUSIONES}

Las unidades de análisis recopiladas hasta el presente informe, han sido analizadas a efectos de poder derivar sus principios o factores subyacentes, posteriormente se ha procedido a agruparlas como se ha indicado en el apartado de metodología.

Cada grupo reúne ejemplares en función del factor discriminado, que posee en común con otras muestras. Asimismo, estas categorías han sido nominadas bajo términos y/o frases referenciales que si bien son arbitrarias, buscan reflejar la esencia característica de los principios aglutinantes mencionados. Las explicaciones de los factores y lo que representan, se encuentran en el apéndice $A$; mientras que las muestras se encuentran en apéndice $C$.

\subsection{Proceso de construcción de los principios heurísticos o factores. Criterios de agrupamiento y categorización. Hallazgos}

Hemos constatado que las heurísticas (o principio heurísticos) construidas a partir de las muestras, no siempre poseen como base la misma dimensión, característica, cualidad o fenómeno.

En algunos casos el elemento que poseen en común diversas muestras (el factor o principio heurístico que las agrupa), esta sustentado en un principio de búsqueda; un ejemplo de esto es el principio al que llamamos: tratamiento de eventos traumáticos y fricciones cotidianas, en este caso el principio apunta a la búsqueda de fricciones en contextos situacionales. En el caso del principio llamado construcciones modulares, el principio no es una búsqueda en un contexto situacional, sino una estructura cualquiera que sea susceptible de ser construida por módulos.

En otros casos, el principio aglutinante es el mercado al que se dirige un producto, negocio o servicio (Ejemplo: desarrollar un servicio con tecnologías anteriores, no de punta; que se dirigen con un precio sustancialmente menor, a mercados marginales que no pueden acceder al producto original). La construcción y/o derivación de un heurístico implica un análisis de diversas dimensiones y niveles, hasta encontrar aquella que mejor refleja el principio de agrupamiento.

\subsection{Combinación de principios}

Se ha verificado que algunas unidades muestrales pueden asignarse a más de un factor simultáneamente, lo que significa que se explican con más de un principio, o lo que es lo mismo: en algunos casos existen diferentes principios combinados.

A modo de ejemplo, La marca Suiza GEBERIT, dedicada a la fabricación de sanitarios, elabora inodoros-bidé suspendidos con la cisterna empotrada detrás de la pared. En el análisis realizado esta innovación, frente a los inodoros y cisterna tradicionales ofrece mayor espacio, lo que encuadra dicha innovación en tres factores orientativos o aplicativos: Eventos simultáneos, decoración funcional, y recursos R.E.I. (Ver apéndice A para explicación de los factores).

Los resultados sobre la simultaneidad de más de un factor fue la siguiente: 67 de las 342 muestras combinan al menos 2 principios; 16 de las muestras combinan tres principios. No se han encontrado muestras que requieran agruparse en una mayor cantidad de principios. 275 muestras (un $80.4 \%$ ) pueden agruparse mediante un solo principio. (ver Tabla 4). 


\subsection{Consideraciones conceptuales}

Hemos advertido que el proceso de discriminación en factores (extracción del factor no sustentado en el estado de la técnica) revela que hay innovaciones cuyos factores orientativos o aplicativos discriminados, evidencian mayor abundancia aplicativa sobre otras estructuras (productos, servicios, ETC.), mientras en otros principios esta característica de potencial aplicativo es más específica.

A modo de ejemplo: el principio Asistencia a Distancia, que agrupa a todos los servicios que, total o parcialmente son provistos sin la presencia del beneficiado o usuario; se presenta con mayores posibilidades evidentes de ser aplicado a diversos servicios, ya que en casi todos ellos existe algún proceso relevante que no requeriría la presencia simultánea de proveedor y usuario.

En otros casos, por ejemplo en el caso del principio denominado Gestión de Acceso Continuo, principio que agrupa a todas las innovaciones que permiten que pueda intervenirse en un proceso sin la necesidad de interrumpirlo (un ejemplo es el lavarropas AdWash de Samsung, que posee una puerta frontal que permite que se introduzca ropa aún cuando el artefacto esta en funcionamiento); las posibilidades de aplicación no se presentan como tan evidentes o abundantes, dado que no en todos los productos, procesos o servicios, la característica de nointerrupción del servicio, se expone como tan obvia o útil. En resumen: existen principios que ofrecen un potencial aplicativo más abarcador, susceptible de aplicarse sobre a una mayor cantidad de fenómenos; mientras que en otros prima una especificidad situacional.

Asimismo, el principio que hemos denominado 4Bios, si bien presenta un elemento aglutinador claro, su modalidad aplicativa no es tan evidente sin antes analizar el contexto de aplicación.

\subsection{Relación de los factores con las líneas y modelos teóricos}

Hemos agrupado los factores encontrados en relación a sus similitudes más evidentes, finalizadno con siete categorías. Asimismo, observamos que existe en algunos casos, una estrecha relacion entre las siete categorías construidas, y algunos de los modelos y perspectivas volcadas en las fundamentaciones teóricas. A continuación se discute dicha relación. Las siete categorías son las siguientes:

1. Innovaciones sustentadas en el eje de mercadotenia o eje mercado-producto.

2. Innovaciones susntadas en la búsqueda de fricciones.

3. innovaciones sustentadas en factores Modulares y de Arquitectura.

4. Innovaciones sustentadas en factores en nuevos formatos de negocios.

5. Innovaciones sustentadas en la modificación de la perspectiva del concepto de recurso.

6. Innovaciones sustentadas en la información, medición e interconexión.

7. Innovaciones sustentadas en la filosofía de frugalidad.

La tabla 4 expone la relación de los factores encontrados, las categorías construidas en función a sus similitudes, y una breve asociación a la literatura más relevante. Debido a la extensión de la tabla, la misma se encuentra disponible en:

https://docs.google.com/document/d/1b7HV3wYtMwdY62T4g9-LI 52Zph21MMnSJJmSFUDYU/edit?usp=sharing 


\subsection{1 - Sustentadas en cambios sobre el eje mercado - producto}

En la tabla 4, el primer grupo reúne los factores encontrados que se relacionan con una perspectiva de mercadotecnia en referencia al eje mercado-producto. De esta forma, los casos que se agrupan dentro del factor Enfoque Fundamental o Etapa (a modo de ejemplo), se refieren a las innovaciones en donde la novedad es la especialización en una etapa de la cadena de valor de una industria.

Otra mención de relevancia es el factor Mercados Marginales y no cualificados, íntimamente relacionados con el concepto de NO CLIENTE del modelo de la Estrategia del Océano Azul.

Esta grupo se relaciona con:

1. Los modelos de marketing tradicionales (especialización, segmentación y nicho).

2. El modelo de innovación en valor de la Estrategia del Océano Azul (Mauborgne R, Kim W, 2005).

Son modelos sustentados en los movimientos de cambios de mercado.

\subsection{2 - Sustentadas en factores relacionados con la búsqueda de fricciones}

En este caso, la perspectiva de la innovación no está sustentado en qué cambios realizar, sino en dónde buscar. Se sustenta en el hecho que buscar fricciones es encontrar focos de potencial valor, ya que permite luego buscar una solución (el concepto de fricción se utiliza aquí como sinónimo de todo aquello que constituye una molestia o inconveniente para el cliente).

Aquí lo novedoso es el tipo de fricción que se busca más que la forma de abordarla. El término puntos de dolor (pain points) se utiliza habitualmente para referirse a este tipo de búsquedas. En este caso, este grupo se relaciona con dos de los modelos tratados en los fundamentos teóricos:

1. El área de búsqueda de potenciales de P. Drucker denominada: La falta de un proceso o el proceso faltante (Drucker $P, 1985$ ). El autor se refiere a la búsqueda de esos inconvenientes para los cuales no hay proceso realizado, por lo que observarlos y detectarlos, es una fuente de potenciales innovaciones.

2. Asimismo, también tiene sustento teórico en La Estrategia del Océano Azul (Mauborgne R, Kim W, 2005), como la exploración de la cadena de compradores.

\subsection{3 - Sustentadas en factores Modulares y de Arquitectura}

Los factores agrupados en dicha categoría, refieren a aquellos que se sustentan en cambios modulares o cambios en la combinación de elementos. Este grupo expone un parentezco con el modelo de Henderson and Clark (1985), en el que presentan cuatro tipos de innovaciones, dentro de las cuales hay dos en que mas relaciones poseen con los factores de dicho grupo: Innovaciones Modulares e Innovaciones de Arquitectura.

\subsection{4-Innovaciones sustentadas en nuevos formatos de negocios}

Aquí se han agrupado los factores que obedecen a cambios en los formatos de negocios tradicionales; se sustentan en supuestos distintos a los que tradicionalmente se habían desarrollado hasta el momento. A modo de ejemplo, el factor Itinerante perteneciante a este 
grupo, refiere a todos los negocios o soluciones que son ambulantes, como ser los camioneshospital de África; los cuales rompen el supuesto de estructura edilicia inmovil.

\subsubsection{Innovaciones sustentadas modificación de perspectiva con la que se percibe lo que es un recurso.}

Este grupo hace referencia a todos los factores cuyas muestras, están sustentadas en el principio de cambio en la forma de ver un recurso. Está íntimamente relacionado con el desarrollo naturalmente sostenible, el cuidado de los recursos, el mayor aprovechamiento de estos, y el hecho de comenzar a considerar como recrusos aquello que antes eran desperdicios o deshechos.

Corresponde a un cambio de perspectiva sobre el mismo concepto de aquello que constituye un recurso, y por lo tanto con nuevos usos.Podemos asociarlo con el pensamiento expuesto por P. Drucker, en la que menciona que la innovación "es la acción de dotar a los recursos de una nueva capacidad de generar riqueza, la innovación crea un recurso" (Drucker P., 1985).

\subsubsection{Innovaciones sustentadas en la información, medición e interconexión.}

Este agrupamiento reúne a las innovaciones que se relacionan con el ámbito de la información y medición sobre los objetos, procesos, recursos, eventos; más que con estos en sí mismo. Los factores o principios agrupados aquí, están en la actualidad relacionados con las nuevas tecnologías de información e internet de las cosas; sin embargo en el grupo de factores mencionados, lo que genera la innovación es la forma en que se utiliza dicha tecnología o sobre que evento, recurso se aplica; no la tecnología en sí misma. Esto es: la innovación se sustenta en una modalidad novedosa de utilizar las tecnologías mencionadas.

Como mencionamos, si bien los principios están actualmente relacionados con las denominadas TICS, su validez es aplicable independientemente del grado de avance de las tecnologías de información y comunicación.

\subsubsection{Innovaciones sustentadas en el concepto FRUGAL Y REVERSE INNOVATION.}

El último grupo, reúne a los factores están íntimamente asociados a la filosofía de innovación desarrollada por N Radjou, J Prabhu en el año 2015; como así también con el concepto de Innovación Inversa (Reverse Innovation) expuesto por Govindarajan Dartmouth en 2009. Los tres factores mencionados en dicho agrupamiento, se refieren a tres formas diferentes que hemos encontrado acerca de cómo se llega al desarrollo de este grupo de innovaciones: por la utilización de principios físicos y químicos fundamentales, esto es: por principios de amplia accesibilidad y conocimiento.

El segundo factor refiere a quitar todas las funcionalidades accesorias a un producto, dejando solo aquella que es esencial, asimismo en lo posible; modificar los materiales de construcción; todo esto con el fin de poder generar innovaciones de carácter frugal o básico, con un acotamiento de costos drástico. Por último, el tercer factor hace referencia a la utilización de la tecnología anterior como vía de solución a otros abordajes más actuales.

\section{CONCLUSIONES}

Durante el proceso de desarrollo del estudio, hemos advertido cuatro aspectos que consideramos que reúnen las observaciones mas relevantes del mismo: en primer lugar (y al igual 
que todo producto con pretención científica), verificar el grado en que los objetivos del estudio fueron alcanzados.

En segundo lugar, ponderar adecuadamente las cualidades de aquello que hemos denominado en el estudio como factores aplicativos $u$ orientativos (los principios resutantes del proceso de discriminación).

En tercer lugar, exponer los que consideramos como ciertas limitaciones encontradas en el trayecto de desarrollo del estudio; y por último: el aspecto contributivo del método utilizado en el estudio, en correlato con lo hallado en la literatura.

Respecto al primer aspecto (el alcance de los objetivos), y comenzando por el objetivo número uno planteado; hemos detectado que todas las muestras analizadas de innovaciones no sustentadas en tecnología de punta, poseen un factor subyacente que les otorga el carácter de novedad y utilidad. Asimismo, respecto al segundo objetivo, se han registrado 50 factores representativos de los 284 casos, 342 casos considerando que existen innovaciones en las que se superponen más de un factor.

Han sido desarrollados siete agrupamientos en función a los cincuenta factores mecionados, realizando un tratamiento de cada uno en el apartado de Resultados y Discusiones. Estos siete agrupamientos, refieren al tercer objetivo: relacionar los mismos con las líneas teóricas expuestas en la literatura. Excepto en el caso de aquellas innovaciones que se sustentan en información, medición e interconexión, los que no se relacionan con un modelo o perspectiva en particular, sino que obedecen a formas novedosas de utilizar las diversas tecnologías para realizar mediciones, utilizar la información e interconexión que estas permiten.

En relación a los objetivos cuatro y cinco: hemos utilizado un método de análisis de cada muestra, que nos ha permitido discriminar y aislar el componente de la dimensión no tecnológica de cada muestra. Lo expresado anteriormente, permite inferir que a partir de dichos principios, y con un aumento de las muestras en el progreso de la investigación; existe una posibilidad cierta de construir métodos aplicativos a efectos de generar innovaciones; sustentado en los factores discriminados y otros que llegasen a detectarse en futuros estudios.

Con respecto al segundo aspecto (la cualidad de los factores aplicativos u orientativos): hemos advertido que existe una relevancia respecto a las cualidades que dichos factores debían atender, y entendemos que en esas observaciones se asienta una de las contribuciones centrales del estudio, y fundamentalmente para futuros estudios. El hecho que un factor (o principio) pueda categorizarse como aplicativo (u orientativo), depende tanto de si permite reducir o acotar el espacio de búsqueda con vistas a generar una innovación; como así también del grado en que reduce dicho espacio.

Cuanto más vasto es el campo (o áreas) en donde realizar la búsqueda, más arduo será el proceso y mayor tiempo involucrará, sin que necesariamente pueda obtenerse el resultado buscado (la generación de una innovación). Asimismo, hemos advertido que si el factor orientativo o aplicativo posee un elevado grado de especificidad, será solo aplicable a casos muy concretos y particulares, perdiendo asimismo su capacidad de extensión o extrapolación a otras áreas (otros productos, industrias, negocios, casos, Etc.). En vistas a lo mencionado, consideramos que hay al menos dos condiciones que deben tenerse en cuenta: el factor debe ser convergente a una solución y simultáneamente, no perder su capacidad de poder extender su utilización en otras áreas (estructuras, productos, negocios, modelos, etc). 
Por otra parte, hemos observado que existe una diferencia conceptual (y fáctica) relevante, entre lo que podemos considerar como factor aplicativo $u$ orientativo por un lado, y una categoría descriptiva y genérica de innovaciones por otro. En la literatura recorrida, los focos de valor o las clases de innovaciones resultantes de los modelos matriciales; se presentan mas como categorías descriptivas de los tipos de innovación (o su fuente), que como principios de capacidad aplicativa u orientativa. Es por esta razón que consideramos la relevancia de las cualidades a las que debe tender la discriminación y posterior construcción de factores aplicativos u orientativos, las cuales para el caso del presente estudio se han advertidos dos: convergencia aplicativa y grado de especificidad.

Respecto al tercer aspecto (limitaciones y obstáculos), hemos advertido algunos puntos que caben ser destacados a modo de contribución, y en vistas a futuros estudios: el primero de ellos es que el grado de aplicabilidad u orientación de los factores que hemos discriminado y desarrollado, es variado; esto es: los factores encontrados, no poseen en todos los casos las cualidades ideales expuestas anteriormente: convergencia aplicativa y grado de especificidad.

Esta variación en la cualidad de convergencia aplicativa se debe fundamentalmente a dos razones, la primera es el tamaño de la muestra: el volumen de unidades muestrales ha sido de utilidad a efectos del objetivo central del estudio, esto es: la verificación de la existencia de factores aplicativosy/o orientativos en las innovaciones no sustentadas en el estado de la técnica.

Sin embargo, algunos casos se han presentado con una menor frecuencia de aparición, el factor denominado como 4 bios mencionado con anterioridad es un ejemplo (este factor refiere a innovaciones sustentadas en el aprovechamiento de las fuerzas, los recursos y las "soluciones" de la naturaleza, a efectos de ser utilizados o extrapolados a problemáticas de la existencia humana, ver apéndice A). Lo anterior resulta en que no existe un suficiente volumen de muestras que haya permitido dotar al factor en cuestión, de mayor convergencia aplicativa.

Para este y otros factores expuestos, hemos considerado que se requiere una mayor cantidad de muestras, a efectos de obtener eventualmente factores más con una mayor cualidad aplicativa. Otro limitante que hemos encontrado, es referido al objeto de estudio en sí mismo, y a su relación a las fuentes de los datos: las cualidades de novedad y utilidad que le confiere a una innovación la designación como tal; no ofrece indica el grado de relevancia de la misma; sin embargo dicha relevancia sí existe en la valoración de los individuos, consumidores o usuarios. Esto es: no todas las innovaciones pueden jerarquizarse como iguales en términos de relevancia.

En función a lo comentado y en relación al estudio, no siempre fue posible a través de las fuentes de datos, advertir dichas dimensiones respecto a cada muestra. Si bien el propio hecho de su difusión en los medios, sumado a la permanencia en el mercado, pueden sugerir medidas indirectas del carácter innovador de una muestra; no fue posible en muchos casos mensurarlas y jerarquizarlas con indicadores más directos y precisos (como pueden ser la tasa de crecimiento de las ventas, el volumen de individuos que incorpora la innovación, o el grado percibido del impacto de la misma).

Por otra parte, colocar exclusivamente el foco en aquellas que sí pueden tratarse con indicadores directos; reduce en gran medida el tamaño muestral debido a la falta de acceso a esa clase de datos, lo cual resta a la riqueza central del estudio: la observación y detección de factores aplicativos u orientativos. A efectos de futuros estudios, se sugiere en primer lugar la priorización del tamaño muestral, conjuntamente a la construcción de indicadores indirectos más eficientes (ante la dificultad de contar con indicadores directos de crecimiento, ventas, incorporación de los usuarios o consumidores, y relevancia). 
Según lo advertido, algunas contribuciones sobre eventuales indicadores indirectos pueden ser: la diversidad de tipos de medios de difusión que se hacen eco de la, la perdurabilidad en el tiempo de la innovación, las diversas regiones geográficas en las cuales la innovación es incorporada o incluso difundida.

Por último, y exponiendo el cuarto aspecto mencionado en el inicio de estas conclusiones (el aspecto contributivo del método utilizado):

Hemos observado en el recorrido de la literatura expuesta; la existencia de una brecha no explorada respecto al estudio de las innovaciones en vistas a la construcción de principios aplicativos más específicos, orientadores, y convergentes. No hemos encontrado en nuestra recopilación estudios que aborden las innovaciones desde un proceso de discriminación de sus componentes no sustentados en el estado de la técnica a efectos de construir posteriormente, factores aplicativos.

Por lo anterior es que entendemos que el procedimiento utilizado de discriminación y posterior derivación de factores se presenta en sí mismo (y adicionalmente a los factores detectados), como un abordaje contributivo y complementario; a ser considerado y continuar explorando; con potencial de ampliación y profundización de cara a futuras investigaciones respecto al estudio del fenómeno de la innovación.

\section{REFERENCIAS}

ABERNATHY W.J., CLARK K.B., Innovation: Mapping the winds of creative destruction, Research Policy., Elsevier., Países Bajos, 14 : 3-22, 1985 https://doi.org/10.1016/0048-7333(85)90021-6.

ALTSHULLER, G.S., Como aprender a Inventar, Fundación de G. S. Altshuller, Rusia, 1961.

BELEN, M.; PEREZ, J. MV M. Metaheurísticas - Una visión global. Revista Iberoamericana de Inteligencia Artificial., Iberamia., España, V. 5, n.19, p. 7-28, 2003. Disponible en: https://www.redalyc.org/pdf/925/92571901.pdf

CHAMBERS Diccionario de Lengua Inglesa - versión de consulta online. Disponible en: https://chambers.co.uk/search/?query=innovate\&title=21st

CHAMBERS Diccionario de Lengua Inglesa - versión de consulta online. https://chambers.co.uk/search/?query=technology\&title=21st.

CHAN, W; MAUBORGNE, R. La estrategia del Océano Azul. Grupo Editorial NORMA. Bogotá, Colombia, 2005.

DRUCKER, P. La innovación y el empresario Innovador. Edhasa. Barcelona, España, 1985. FREEMAN, C. The economics of technical change, Cambridge Journal of Economics, Volume 18, Issue 5, October 1994, Pages 463-514, https://doi.org/10.1093/oxfordjournals.cje.a035286

GEE, S (1981) Technology transfer, Innovation \& International Competitiveness. Wiley and Sons. New York, 1981. 
GODIN, B. (2008). Innovation: the history of a category Working Paper. Institut national de la recherche scientifique, Centre Urbanisation Culture Société, Montréal. Disponible en: http://espace.inrs.ca/id/eprint/10023/1/Godin_2008.pdf

GOLDENBERG, J. Finding your innovation sweet spot. Harvard Business Review, E.E.U.U., 2003. Disponible en: https://hbr.org/2003/03/finding-your-innovation-sweet-spot

GOVINDARAJAN, V.; TRIMBLE, C. Reverse Innovation: Create far from home, win everywhere. Harvard Business School Press, 2012

HATZICHRONOGLOU, T. Revision of the high-technology sector and product classification. OCDESTI working papers, 1992. Disponible en: https://read.oecd-ilibrary.org/science-andtechnology/revision-of-the-high-technology-sector-and-productclassification_134337307632\#page2

HENDERSON, R; CLARK, K, Architectural Innovation: The Reconfiguration of Existing Product Technologies and the Failure of Established Firms. Administrative Science Quarterly, 1990. http://dx.doi.org/10.2307/2393549. Disponible en: https://www.researchgate.net/publication/200465578_Architectural_Innovation_The_Reconfigur ation_of_Existing_Product_Technologies_and_the_Failure_of_Established_Firms

KOTSEMIR, M.; ABROSKIN, A. Innovation Concepts and Typology - An Evolutionary Discussion. National Research University Higher School of Economics, Universidad de Munich, 2013 n. 45069 Working Paper BRP 05/STI/2013. Disponible en: https://mpra.ub.unimuenchen.de/45069/1/MPRA_paper_45069.pdf

LEWIS, M. The New New Thing W. W. Norton \& Company, E.E.U.U., 1998.

OECD Frascati Manual - Guidelines for Collecting and Reporting Data on Research and Experimental Development. OECD, Septima edición, 2015. Disponible en https://read.oecdilibrary.org/science-and-technology/frascati-manual-2015_9789264239012-en, https://doi.org/10.1787/24132764

OECD Oslo Manual - Guidelines for Collecting and Interpreting Innovation data. OECD, Tercera edición, 2005. Disponible en: https://read.oecd-ilibrary.org/science-and-technology/oslomanual_9789264013100-en, https://doi.org/10.1787/19900414

OECD Oslo Manual - Guidelines for Collecting and Interpreting Innovation data. OECD, Cuarta edición, 2018. Disponible en: https://read.oecd-ilibrary.org/science-and-technology/oslo-manual2018_9789264304604-en, https://doi.org/10.1787/24132764

OECD. Products Set to Sizzle, versión online, OCDE Aid for Trade, 2011 Disponible en: https://www.oecd.org/aidfortrade/48368557.pdf, https://doi.org/10.1787/22234411 RUEDA, F.G.; VERGER, F, OECD Taxonomy of Economic Activities Based on R\&D Intensity, 2016, https://doi.org/10.1787/18151965 
OECD Science, Technology and Industry Scoreboard. Disponible en:

https://www.oecd.org/sti/scoreboard.htm

OVANS, A. What Is a Business Model?, Harvard Business Review, Enero 2015.

PEREZ, C, Technological Revolutions and Techno-economic paradigms, Cambridge Journal of Economics, Vol. 34, No.1, pp. 185-202, 2010. https://doi.org/10.1093/cje/bep051

RAE Diccionario de la Real Academia Española - versión de consulta online.

https://dle.rae.es/innovaci\%C3\%B3n?m=form

RADJOU, N.; PRABHU, J. Frugal Innovation: How to Do More with Less. The Economist, 2015

ROUSE, W. Strategies for Innovation. Wiley-Interscience, John Wiley and Sons, Inc., Nueva York, 1992, v. 39, n. 2, p. 201-203. https://doi.org/10.1080/00137919408945392

SCHRIJVER, A. On the history of combinatorial optimization (till 1960) - Handbook, Volumen $12 \mathrm{p}$. 1-68, Handbook. Elsevier, 2005, https://doi.org/10.1016/S0927-0507(05)12001-5

SCHUMPETER, J. Business Cycles: A Theoretical, Historical, and Statistical Analysis of the Capitalist Process. McGraw-hill, New. York and London, 1939. 


\section{APÉNDICE A - DESCRIPCIÓN Y EXPLICACIÓN DE LOS FACTORES}

Dada la extensión de la Tabla 5, se facilita a continuación un link para su visualización. Disponible en:

https://docs.google.com/document/d/1vaUpSh7XCvxsqcyaC4DTkJJjXt74kFSsLOYjmr8vOz4/edit?us p=sharing

\section{APÉNDICE B - EJEMPLOS DE CADA FACTOR ORIENTATIVO - APLICATIVO DISCRIMINADO}

Dada la extensión de la Tabla 6, se facilita a continuación un link para su visualización. Disponible en:

https://docs.google.com/document/d/1vaUpSh7XCvxsqcyaC4DTkJJjXt74kFSsLOYjmr8vOz4/edit?us $\mathrm{p}=$ sharing

\section{APÉNDICE C - CONJUNTO DE LAS MUESTRAS}

Dada la extensión de la Tabla 7, se facilita a continuación un link para su visualización. Disponible en:

https://docs.google.com/document/d/1wCZw9CsmhYZ3KdNebuc-1jh1FfgHfvm3ZcsufM5fSs/edit?usp=sharing

${ }^{1}$ Uma versão reduzida deste artigo foi publicado no VI Congresso Internacional da Rede ACINNET 2020.

${ }^{1}$ A shortened version of this paper was published at the VI ACINNET Network Conference 2020. 